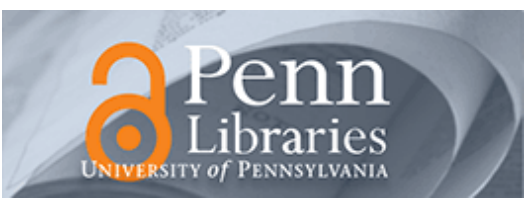

University of Pennsylvania

ScholarlyCommons

Departmental Papers (CBE)

Department of Chemical \& Biomolecular

Engineering

10-10-2004

\title{
Surface probe measurements of the elasticity of sectioned tissue, thin gels and polyelectrolyte multilayer films : correlations between substrate stiffness and cell adhesion
}

\author{
Adam J. Engler \\ University of Pennsylvania, aengler@seas.upenn.edu \\ Ludovic Richert \\ Université Louis Pasteur \\ Joyce Wong \\ Boston University \\ Catherine Picart \\ Université Louis Pasteur \\ Dennis E. Discher \\ University of Pennsylvania, discher@seas.upenn.edu
}

Follow this and additional works at: https://repository.upenn.edu/cbe_papers

Part of the Biochemical and Biomolecular Engineering Commons

\section{Recommended Citation}

Engler, A. J., Richert, L., Wong, J., Picart, C., \& Discher, D. E. (2004). Surface probe measurements of the elasticity of sectioned tissue, thin gels and polyelectrolyte multilayer films : correlations between substrate stiffness and cell adhesion. Retrieved from https://repository.upenn.edu/cbe_papers/26

Postprint version. Published in Surface Science, Volume 570, Issues 1-2, 10 October 2004, pages 142-154.

Publisher URL: http://dx.doi.org/10.1016/j.susc.2004.06.179

This paper is posted at ScholarlyCommons. https://repository.upenn.edu/cbe_papers/26

For more information, please contact repository@pobox.upenn.edu. 


\title{
Surface probe measurements of the elasticity of sectioned tissue, thin gels and polyelectrolyte multilayer films : correlations between substrate stiffness and cell adhesion
}

\begin{abstract}
Surface probe measurements of the elasticity of thin-film matrices as well as biological samples prove generally important to understanding cell attachment across such systems. To illustrate this, sectioned arteries were probed by Atomic Force Microscopy (AFM) within the smooth muscle cell (SMC)-rich medial layer, yielding an apparent Young's modulus $E_{\text {media }} \sim 5-8 \mathrm{kPa}$. Polyacrylamide gels with $E_{\text {gel }}$ spanning several-fold above and below this range were then cast 5-70 $\mu \mathrm{m}$ thick and coated with collagen: SMC spreading shows a hyperbolic dependence in projected cell area versus $E_{\text {gel }}$. The modulus that gives halfmax spreading is $E_{1 / 2}$-spread $\sim 8-10 \mathrm{kPa}$, proving remarkably close to $E_{\text {media. }}$. More complex, layer-by-layer micro-films of poly(L-lysine)/hyaluronic acid were also tested and show equivalent trends of increased SMC spreading with increased stiffness. Adhesive spreading of cells thus seems to correlate broadly with the effective stiffness of synthetic materials and tissues.
\end{abstract}

\section{Keywords}

Substrate compliance, Polyelectrolyte multilayers, Surface structure, Surface morphology, Surface roughness, Surface topography, Adhesion, Atomic force microscopy

\section{Disciplines}

Biochemical and Biomolecular Engineering

\section{Comments}

Postprint version. Published in Surface Science, Volume 570, Issues 1-2, 10 October 2004, pages 142-154. Publisher URL: http://dx.doi.org/10.1016/j.susc.2004.06.179 


\title{
Surface probe measurements of the elasticity of sectioned tissue, thin gels and polyelectrolyte multilayer films: correlations between substrate stiffness and cell adhesion
}

\author{
Adam Engler*, Ludovic Richert ${ }^{\#}$, Joyce Wong ${ }^{\dagger}$, Catherine Picart ${ }^{\#}$, and Dennis Discher*§ \\ *Biophysical Engineering Lab, Mechanical Engineering \& Applied Mechanics and Chemical \& Biomolecular \\ Engineering; University of Pennsylvania; Philadelphia, PA 19104 \\ \#Institut National de la Santé et de la Recherche Médicale, Unité 595, Université Louis Pasteur, 11 Rue \\ Humann, 67085 Strasbourg Cedex, France \\ ${ }^{\dagger}$ Department of Biomedical Engineering; 44 Cummington St.; Boston University; Boston, MA 02215 \\ ${ }^{\S}$ Corresponding author: 112 Towne Building/220 S. 33 ${ }^{\text {rd }}$ St. $\quad$ discher@seas.upenn.edu \\ Philadelphia, PA $19104 \quad$ (ph.) 215-898-4809, (fax) 215-573-6334
}

\begin{abstract}
Surface probe measurements of the elasticity of thin-film matrices as well as biological samples prove generally important to understanding cell attachment across such systems. To illustrate this, sectioned arteries were probed by Atomic Force Microscopy (AFM) within the smooth muscle cell (SMC)-rich medial layer, yielding an apparent Young's modulus $E_{\text {media }} \sim 5-8 \mathrm{kPa}$. Polyacrylamide gels with $E_{g e l}$ spanning several-fold above and below this range were then cast $5-70 \mu \mathrm{m}$ thick and coated with collagen: SMC spreading shows a hyperbolic dependence in projected cell area versus $E_{g e l}$. The modulus that gives half-max spreading is $E_{1 / 2 \text {-spread }} \sim 8-10 \mathrm{kPa}$, proving remarkably close to $E_{\text {media. }}$. More complex, layer-by-layer micro-films of poly(L-lysine)/hyaluronic acid were also tested and show equivalent trends of increased SMC spreading with increased stiffness. Adhesive spreading of cells thus seems to correlate broadly with the effective stiffness of synthetic materials and tissues.
\end{abstract}

Running Title: Film Elasticity vs. Cell Response

Abbreviations: PA, polyacrylamide; AFM, atomic force microscopy; PLL, Poly(L-lysine); HA, Hyaluronic Acid; SMC, smooth muscle cell; DMEM, Dulbeco's Modified Eagle Medium

Keywords: Substrate Compliance, Adhesion, Polyelectrolyte Multilayers, Atomic Force Microscopy 
Surface Science - Revised Manuscript

\section{Introduction}

As a surface probe method for local elasticity measurements, Atomic Force Microscopy (AFM) has been widely used on many materials, both synthetic and natural. In growing need of such characterizations are thin and soft gels that are now increasingly used in cell culture. Motivation for probing matrix mechanical properties by AFM derives from the relatively recent idea that the elastic or viscoelastic resistance of a substrate to cell-generated forces is intrinsic to a cell's in vitro response, from adhesive spreading to differentiation (1-4). In other words, a cell is tactile in attaching and responding to a ligand-coated surface - it is not simply a droplet that spreads on a favorable surface. 'Rheobiology' aptly describes the phenomena while suggesting tissuelevel mechanisms. Accurate and arguably highly local measurements of matrix and substrate mechanical properties are thus increasingly needed for understanding cell responses on surfaces that might or might not mimic a particular tissue's elasticity.

Pelham and Wang (3) were the first to grow cells on collagen-coated polyacrylamide (PA) gel substrates of varied elasticity. Adjusting the gel's crosslinker, bis-acrylamide, permits systematic observation of elasticity-dependent differences in cell behavior. Cell spreading, cell crawling, and focal adhesion assembly are a few of many cell responses found to be influenced by such elasticity manipulations (1-6). It has become clear, however, that various elastic moduli for $\sim 100 \mu \mathrm{m}$ thick PA gels reported in Pelham and Wang (3) and Lo et al. (2) are incorrect by up to orders of magnitude when compared to more recent reports on PA gel elasticity $(1 ; 4 ; 7)$. Accurate measurements of PA gel elasticity, particularly as measured here by AFM, allow comparisons with tissue properties and highlight the need for systematic assessments of cell response together 
with matrix elasticity, where length scale effects normal to the substrate are also considered together with lateral accuracy.

AFM is a useful surface probe method not only for probing cell culture substrates but also for probing the elasticity of cells and perhaps even tissues that previously have been studied only by macroscopic methods (8). Cells in vitro have been reported to have effective Young's moduli, $E_{\text {cell, }}$, in the range of $1-100 \mathrm{kPa}$ (8-12). Relevant here, this range encompasses measurements of elasticity found for vascular smooth muscle cells (SMC) in culture, motivating questions of arterial media elasticity. Blood vessels, particularly arteries with complex structure and function, provide good examples of the possible insight to be gained from probing vascular cross-sections with three distinct annular layers of very different composition (Fig. 1). The medial layer typically consists of $\sim 30-60 \%$ SMCs and is also rich in collagen $(\sim 10-40 \%)$ as well as elastin, which is why the media is often associated with vessel wall elasticity. However, even with the complexity of the mechanical environment for these cells, a single composite modulus (eg. axial stress / vessel's extensional strain) of pressurized vessels in high strain regimes is typically all that is reported or known (8) to represent both static and dynamic elastic loading situations. The arterial media is predominantly smooth muscle cell (SMC) tissue, with a Young's modulus $E_{\text {media }}$ that can presently only be estimated. For example, dog ureter muscle, when relaxed, has a modulus in the range of 5-20 $\mathrm{kPa}$ at moderate strains of $10-30 \%(8 ; 9)$. Within this seemingly narrow range of stiffness, adhesive spreading of vascular SMC is known to vary greatly in vitro (1).

If understood well enough, the layered geometry of arterial vessel walls or perhaps just the elastin layering within the media might be effectively mimicked with 
multilayer films of alternating polyelectrolytes. Polyelectrolyte multilayer films made by the layer-by-layer method of alternately dipping a substrate in cationic and anionic polyelectrolyte solutions $(13 ; 14)$ create a highly controllable system dominated by electrostatic interaction between layers (analogous to the coupling between layers of SMCs in the arterial vessel wall). While serum deposition is known to promote cell adhesion in nanometer thin and dense films, such deposition has little effect on swollen films $(15 ; 16)$. However, very little is yet known about the elasticity of multilayer films, including effects of layer number, thickness, and type of polyelectrolyte, as well as the influence of film elasticity on cell adhesion.

As a representative study in surface probe measurements from in vivo to in vitro, a specific goal here is to clarify the mechanical environment seen by SMC in vivo and to begin correlating this with SMC responses on collagen-coated polymer matrices. Here, length scale effects relevant to SMC sensing of matrix stiffness are partially addressed by attention to thickness $h$ of gels and multilayers as well as AFM indentation depth. Lateral sensitivity and accuracy, at least in AFM, are also partly addressed by probing with pyramidal nano-tips versus micro-bead tips and by studying lateral gradients of elasticity. Beyond these technical issues, a key question is whether model matrix materials other than PA gels show similar trends in cell response versus elasticity. To this end, the effective elasticity of layer-by-layer polyelectrolyte films - both with and without covalent crosslinking - are reported together with spreading of SMC on these hin films. Ultimately, it is found that cells spread increasingly on stiffer substrates regardless of height $(>\mu \mathrm{m})$ and composition, with half maximum spreading coincident with tissue elasticity. 


\section{Experimental Procedures}

\subsection{Gel Substrates.}

PA gel substrates were polymerized as homogenous or gradient thin films on rigid glass supports. Gels with homogenous bis-acrylamide crosslinker concentration were prepared per Wang and Pelham (17), with the addition of glass spacer beads of desired diameter. To induce crosslinking, $1 / 200$ volume of $10 \%$ ammonium persulfate and 1/2000 volume of $\mathrm{N}, \mathrm{N}, \mathrm{N}^{\prime}, \mathrm{N}^{\prime}$-tetramethylethylenediamine were added to PA gel solutions, composed of $5 \%$ acrylamide monomer, $0.03-0.3 \%$ bis-acrylamide crosslinker, and $1 \%$ bead spacers (5 - $10 \mu \mathrm{m}$; Duke Scientific; Palo Alto, CA). $25 \mu \mathrm{L}$ drops of polymerizing gel solution were placed on glutaraldehyde-treated aminosilanized coverslips, which covalently bound the PA. Chlorosilanized coverslips were placed on top of the PA, and weights were added to ensure that the gel thickness was defined by the spacer bead diameter. The droplet volume was also used to control gel height. Gel heights were visually confirmed by calibrated through focusing in light microscopy, and AFM was employed to determine the gel stiffness (detailed below). Rat-tail collagen type 1 (BD Biosciences; Bedford, MA) was chemically crosslinked using a heterobifunctional photoactivating crosslinker, sulfo-SANPAH (Pierce, Rockford, IL) per Wang and Pelham (17). Substrate attachment of collagen was measured with 10:1 collagen/FITC-collagen mixture and compared to standard slides of known fluorescent collagen $(1,4)$ in order to calibrate the amount of

surface-bond collagen. Attachment and accessibility to cells was also assessed using a polyclonal anti-collagen antibody that only binds native collagen (Chemicon; Temecula, CA) and quantum dots (Quantum Dot; Hayward, CA) labeled with secondary antibodies. 
To create elasticity gradients within PA gels, a microfluidic method was used $(\mathrm{N}$. Zaari, P. Rajagopalan, S.K. Kim, A. Engler, and J.Y. Wong, in preparation). Briefly, polyacrylamide was photopolymerized in mixing channels patterned in polydimethylsiloxane (PDMS) with two crosslinker inputs $(0.048 \%$ and $0.48 \%$ bisacrylamide) that combined with $8 \%$ acrylamide solutions in the microfluidic channels to create the gradient. The PA gradient gels were $\sim 90 \mu \mathrm{m}$ thick, as confirmed by light microscopy, and were prepared on aminosilanized coverslips.

\subsection{Polyelectrolyte Multilayer Preparation.}

Poly(L-lysine)/Hyaluronic Acid (PLL/HA) multilayer films, where $i$ is the number of layer pairs, were previously shown to be micrometer thick films (18) that could be used as cell substrate for cell adhesion $(15 ; 19)$. They were prepared here by sequential layering on $24 \mathrm{~mm}$ x $24 \mathrm{~mm}$ coverglass cleaned with $10 \mathrm{mM}$ sodium dodecyl sulfate (SDS) and $0.1 \mathrm{~N} \mathrm{HCl}(10 \mathrm{~min})$ and then rinsed with deionized water. PLL (30 kDa), PLL-fluorescein (0.003:0.01 mol fluorescein per mol lysine monomer), and HA (400 kDa; Bioiberica, Spain) were prepared by direct dissolution in filtered $0.15 \mathrm{M} \mathrm{NaCl}$ solution ( $\mathrm{pH}$ 6) at $1 \mathrm{mg} / \mathrm{ml}$ final concentration. Films were prepared by an automatic dipping machine (Dipping Robot DR3, Kirstein GmbH, Germany) in which the glass slides were arranged vertically in a homemade holder and immersed for $10 \mathrm{~min}$ in a first polyelectrolyte solution (PLL). The slides were subsequently rinsed successively in three different solution volumes of $0.15 \mathrm{M} \mathrm{NaCl}(\mathrm{pH}$ ), $350 \mathrm{~mL}, 200 \mathrm{~mL}$ and $200 \mathrm{~mL}$ for 1 min, $2.5 \mathrm{~min}$, and $2.5 \mathrm{~min}$ respectively. Samples were dipped in oppositely charged polyelectrolyte solution, rinsed using the same procedure, and the process was repeated. Rinsing solutions were changed after the deposition of every twentieth layer. Films were 
stored in $0.15 \mathrm{M} \mathrm{NaCl}$ at $4^{\circ} \mathrm{C}$. The thickness of the films was determined by confocal microscopy by measuring the green band in $\mathrm{z}$-sections corresponding to the diffuse band of PLL-fluorescein within the (PLL/HA) films (18).

To covalently crosslink carboxylate and primary amine groups within the film, $400 \mathrm{mM}$ 1-ethyl-3-(3-dimethylamino-propyl) carbodiimide (EDC) and $100 \mathrm{mM} \mathrm{N}$ hydrosulfo-succinimide (Sulfo-NHS) were mixed (v/v) in $0.15 \mathrm{M} \mathrm{NaCl}$ solution (pH 5) (20). The (PLL/HA) $)_{20}$ films and EDC/sulfo-NHS solution were incubated for 12 hours, and rinsing was performed three times with a $0.15 \mathrm{M} \mathrm{NaCl}$ solution for one hour (19).

Collagen was adsorbed to the HA surface of multilayers, whether the PLL/HA film was crosslinked or not, overnight at $37^{\circ} \mathrm{C}$. Excess collagen was rinsed away with $0.15 \mathrm{M} \mathrm{NaCl}$ and substrate attachment was measured using 10:1 collagen/FITC-collagen mixture and comparison to fluorescent collagen standards as above (1, 4). Z-section confocal microscopy was performed to confirm surface localization of collagen on the native and crosslinked PLL/HA multilayers, and fluorescence recovery after photobleaching (FRAP) was used to assess collagen mobility.

\subsection{Tissue Sample Preparation}

Whole carotid arteries were excised from 6 mo. old pigs and studied within 8 hrs. An artery was cut into thin cross-sections with razor blades. The vessel sections were mounted on a coverglass so that the middle-layer - the media where smooth muscle cells (SMCs) are located (see Fig.1) - was exposed for probing by AFM. Tissue samples were kept in Dulbeco's Modified Eagle Medium (DMEM) supplemented with antibiotics. Tissue section thickness was confirmed by light microscopy to be $\sim 100 \mu \mathrm{m}$. Samples were placed in the AFM and indented by a sphere-tipped probe (detailed below). 


\subsection{Cell Culture.}

A7r5 SMCs (aorta-derived cell line) were maintained in polystyrene flasks between passages 2 and 15, and cultured in media containing DMEM, supplemented with $10 \%$ of fetal bovine serum and antibiotics. Cells were passed every 3 days, when reaching $\sim 80 \%$ confluency, and plated on various substrates at $\sim 1 \times 10^{4}$ cells/dish for experiments. Substrates were either glass-bottom $35 \mathrm{~mm}$ dishes (Mattek Corporation; Ashland, MA) or coverslips coated with the relevant thin films. Cell images were taken after 4 hours of incubation and fixation with a $10 \%$ formaldehyde solution and mounting media (Biomeda; Foster City, CA).

Cell culture supplies were from Invitrogen, Life Technologies (Carlsbad, CA); all other supplies were from Sigma (St. Louis, MO) unless noted. Image analysis of projected cell area was performed with Scion Image (Frederick, MD), and is displayed as an average ( \pm standard error of the mean) with paired t-tests to determine significance.

\subsection{Atomic Force Microscopy Characterization of Films and Gels.}

Force - indentation profiles of arterial sections, PA gels, and (PLL/HA) films were collected with an Asylum 1D AFM (Asylum Research; Santa Barbara, CA). Thick homogeneous gels as well as gradient gels, which both range between $70-90 \mu \mathrm{m}$ in thickness, were tested in the AFM with pyramid-tipped cantilevers (Veeco; Santa Barbara, CA) having a nominal spring constant of $\sim 60 \mathrm{pN} / \mathrm{nm}$ that was more precisely determined from thermal calibration. Force - indentation curves obtained with this tip geometry were fit up to $2 \mu \mathrm{m}$ tip deflections with a Hertz cone model $(10 ; 21)$. In addition, homogeneous PA gels of all heights were tested with borosilicate sphere-tipped cantilevers (radius of $2.5 \mu \mathrm{m}$; Bioforce Nanoscience; Ames, IA) having a similar spring 
constant $\sim 60 \mathrm{pN} / \mathrm{nm}$. Force-indentation profiles from these samples were fit with a Hertz sphere model using a thin film correction appropriate when $h=\sim 13 R$, where $h$ is sample thickness and $R$ is the radius of the sphere-tipped indenter (22). In this limit, the importance of a thickness correction and larger contact area increases exponentially relative to the semi-infinite substrate usually assumed for Hertz cone and sphere models (21; 23-25). There was no statistical difference between the elastic moduli determined by the two probe geometries $(\mathrm{p}=0.45)$ on thick PA gels. Crosslinked and non-crosslinked (PLL/HA) films were tested with the same borosilicate sphere-tipped cantilevers $(R=2.5$ $\mu \mathrm{m})$ using the thin film corrected Hertz sphere model. Arterial tissue sections were tested with both cantilever geometries and were sufficiently thick to fit uncorrected versions of the Hertz cone (21) and sphere (23). Samples were indented at rates of approximately 2 $\mu \mathrm{m} / \mathrm{sec}$, which is generally sufficient to explore elastic rather than viscoelastic properties of cells, matrix, or substrates (26).

\section{RESULTS}

\subsection{Tissue Elasticity by Surface Probing: Young's Modulus of Arterial Media}

Cells, their protrusions, and cell-scale extracellular matrix establish dimensions of microns that AFM cantilevers can generally probe with micron-diameter spheres glued to their tips. These probes, as well as more conventional pyramidal tips, can investigate a range of scales down to molecular (i.e. nanometers). With tissue, the combination of length scale and soft substrates motivates AFM characterization using a range of indenter geometries. 
To probe the elasticity within the arterial media, fresh arteries ( 2 $\mathrm{mm}$ diameter) were sectioned into $\sim 100 \mu \mathrm{m}$ long segments (see Methods) and mounted wet under either sphere- or pyramid-tipped AFM cantilevers. Arterial sections $(n=3)$ were probed with approximately ten force-indentation curves each. The curves were fit well up to indentations of $\sim R(2.5 \mu \mathrm{m})$ by conventional Hertz cone (21) and sphere (22) models. Assuming incompressibility, Table 1 lists the fitted modulus within the narrow range $E_{\text {media }}=5-8 \mathrm{kPa}$, appearing almost independent of tip length scale.

Indentation by AFM strains the surface as well as the thickness of the material, with the strain decaying both laterally and with depth. A simple, first estimate of the average radial strain at the surface, $u_{\mathrm{rr}}$, upon indenting to a depth $R$ is readily made for the sphere-tipped cantilever. The material under the spherical indenter's diameter $(2 R)$ is assumed to stretch homogeneously, upon indentation, to half the circumference $(\pi R)$. This gives $u_{\mathrm{rr}} \sim(\pi / 2-1) \approx 55 \%$, which is not extremely large in the context of sustainable arterial strains. Moreover, such a strain exceeds by about two-fold or more the maximum lateral strains of $10-30 \%$ typically generated by cell tractions on a range of soft substrates $(2 ; 27)$.

\subsection{Thin Gel, Elastic Tissue Mimics}

Polyacrylamide (PA) gels, with variable bis-crosslinker and surface-attached collagen, have emerged as a reproducible system for cell studies on the effect of matrix compliance. Most such studies have used PA gels approaching $\sim 100 \mu \mathrm{m}$ in thickness. Much thinner PA gels (Fig. 2A) were obtained by addition of a small volume of spacer microbeads to PA prior to polymerization between two coverslips. Removal of the 
upper, chlorosilanized coverslip yields a gel that is not only covalently bound to the lower coverslip but is also smooth over its top surface. Even for the thinnest gels of 5 $\mu \mathrm{m}$, indentation to a depth $-R(2-3 \mu \mathrm{m})$ with sphere-tipped AFM probes (Fig. 2B) show force profiles in surprisingly good agreement with the Hertz sphere model provided use is made of a thin film correction (22). Without the correction, the classic Hertz model analysis leads to an artificially high modulus (by up to $\sim 50 \%$ here, Fig. $2 \mathrm{C}$ open points), as expected of stress-stiffening with strain constraints at the underlying rigid glass. With the correction, gel elasticity did not vary significantly with film thickness $(p=0.38)$ for either lightly crosslinked $(0.03 \%$ bis $)$ or densely crosslinked $(0.3 \%$ bis $)$ samples, for which respective average moduli are $E_{g e l}=1.4 \pm 0.1 \mathrm{kPa}$ and $8.1 \pm 0.1 \mathrm{kPa}$ (Fig. 2C).

On the thickest, $70 \mu \mathrm{m}$ PA gels, pyramid-tipped probes gave elastic moduli that were not significantly different $(\mathrm{p}=0.45)$ from those determined by the sphere-tipped probes, with or without a correction for sample thickness. Thicker gels also tested by simple tension tests give similar moduli (1). The results here collectively suggest that thin films of soft PA gels $(E \sim 1 \mathrm{kPa})$ as well as moderately stiff PA gels $(E \sim 8 \mathrm{kPa})$ can accurately mimic ECM stiffness without overwhelming stiffening due to the underlying glass substrate.

\subsection{Cell Spreading on Thin Gels.}

Attaching collagen to PA gels creates a more adhesive surface for cells without altering gel elasticity (1). Four hours after plating SMCs (the aorta-derived cell line, A7R5) on various collagen-coated PA substrates, SMCs are more spread on stiffer substrates (Fig. 3A). This substrate-stiffness dependent trend with projected cell area is 
most prominent and clear at low elastic modulus, $E_{g e l}<5 \mathrm{kPa}$. At high $E$, including an extrapolation to cells plated directly on glass, spread cell area plateaus.

Cell spreading is remarkably independent of gel height, $h$; cell spreading on both 5 and $70 \mu \mathrm{m}$ PA gels prove statistically similar $(\mathrm{p}=0.41$, Fig. 3A). Although these cells typically have lateral dimensions of $\sim 50 \mu \mathrm{m}$ or more, traction forces $(27 ; 28)$ are such that cells do not "feel" a rigid substrate that is as close as $5 \mu \mathrm{m}$ away, which is more typical of a cell height than a lateral dimension. On the other hand, modulus determinations by AFM on $5 \mu \mathrm{m}$ substrates clearly require a correction for film thickness. For tissue layers such as the arterial media, however, sensitivity to the local micro-elasticity means that adjacent layers (eg. adventitia or intima) can be stiffer or softer without propagating deeply into a given layer.

The thickness independence down to $h \sim 5 \mu \mathrm{m}$ is sensible since cell tractions have been shown to create lateral displacements of $2 \mu \mathrm{m}$ or less on various PA gels $(27 ; 28)$. The minimum length scale for cell traction propagation normal to the spreading axes (or axis) is an interesting question to address with thinner gels - perhaps together with assessing sensitivity to lateral gradients. The biologically relevant results here seem consistent with relatively homogeneous elastic properties within the media as revealed by the small coefficient of variation in $E_{\text {media }}$ (see Table 1 ).

A hyperbolic fit of cell spreading versus substrate stiffness (1) proves insightful and has a form that defines the half-saturation constant, $E_{1 / 2 \text {-spread: }}$

$$
\text { Area }=a\left(E_{\text {gel }}\right)^{\mathrm{m}} /\left[\left(E_{1 / 2 \text {-spread }}\right)^{\mathrm{m}}+\left(E_{\text {gel }}\right)^{\mathrm{m}}\right]+\text { const. } \quad(m \approx 1)(\text { Eqn. } 1)
$$


For $5 \mu \mathrm{m}$ and $70 \mu \mathrm{m}$ PA gels, $E_{1 / 2 \text {-spread }}=7.9 \mathrm{kPa}$ and $10 \mathrm{kPa}$, respectively; the statistical equivalence of the two datasets thus implies $E_{1 / 2 \text {-spread }} \approx 8.9 \pm 1.1 \mathrm{kPa}$. Most important from this analysis is the remarkable correspondence $E_{1 / 2 \text {-spread }} \approx E_{\text {media }}$ has a physiological interpretation as an elastic set point for SMC. Cells will spread more than normal for substrate perturbations that lead to matrix rigidification, i.e. $E>E_{1 / 2 \text {-spread }}$ (Fig. 3A, large inset cell). Likewise, cells will spread less for substrate perturbations that lead to matrix softening, i.e. $E<E_{1 / 2 \text {-spread }}$ (Fig. 3A, small inset cell).

Adhesive spreading is also dependent on ligand density, hence the need to attach collagen to the surface of gels $(1 ; 4 ; 6)$ for cell receptors to bind. Importantly, gelattached collagen is surface accessible on both supramolecular and micron length scales that are typical of cell receptors and protrusions. This is shown with anti-collagen functionalized quantum dots $(\sim 10 \mathrm{~nm})$ here (see Fig. 3B inset) as well as micron-sized beads (2) that bind only to the gel surfaces when collagen is attached. Addition of functionalized quantum dots after cells are allowed to attach to the gels shows cells exclude the particles.

The coupled dependence of cell spreading on both ligand density and stiffness proves highly non-linear, with a modest but clear maximum in cell spreading on the PA gel substrates (Fig. 3B). At very low ligand on any substrate investigated, SMC spread minimally. On gels with the highest ligand densities, cell spreading is suppressed, especially on soft substrates. Moderate ligand densities $\left(\sim 200 \mathrm{ng} / \mathrm{cm}^{2}\right.$ collagen) lead to considerable cell spreading $(1 ; 4)$. It is important to note that cell spreading in Fig. $3 \mathrm{~A}$ is performed at the optimal collagen density for the given substrates. 


\subsection{Layer-by-Layer Films: Elasticity and Crosslinking}

Multilayers of PLL as the polycation alternating with HA as the polyanion were prepared on glass coverslips or slides with 20 layer pairs, i.e. (PLL/HA) 20 . By use of fluorescein-PLL and confocal microscopy (18), a film thickness of $\sim 4 \mu \mathrm{m}$ was estimated. Samples were then mounted in the AFM and probed with sphere-tipped cantilevers using instrument parameters identical to the probing of PA gels, i.e. indentation rates of $\sim 2 \mu \mathrm{m} / \mathrm{sec}$ and indentation distances of several microns. Force indentation curves of $(\mathrm{PLL} / \mathrm{HA})_{20}$ films were fit with the same thin film correction of the Hertz sphere model (22) used earlier on thin PA gels: ignoring the correction here leads to a similar overestimate as seen with PA gels (Fig. 2C). Importantly, the measurements on multilayers yield an apparent elastic modulus in the $z$-direction, $E_{\text {perp. }}$ The qualifications on this modulus arise in part because the layer-by-layer formation process leads to orthotropic rather than isotropic symmetry, hence measurements perpendicular to the layer symmetry give only a modulus in that direction. Additionally, the PLL layers (but not the HA layers) have been found to reorganize as fluorescent-PLL diffuses slowly throughout the film $(18 ; 19) . \quad E_{\text {perp }}$ for PLL/HA films is thus an apparent modulus with an implicit time scale.

With the above qualifications for the multilayers, (PLL/HA) 20 was determined to have an average $E_{\text {perp }} \sim 85 \mathrm{kPa}$. This appears significantly stiffer than equivalently thick PA gels, and yet the multilayer films here contain highly swellable polysaccharides (eg. HA) and are thus also hydrogels. Differences in chemistry, however, alter pore size, hydration, and crosslinking between monomer units, and thus give very different properties. 
Surface Science - Revised Manuscript

Covalent crosslinking between multilayers should limit polyelectrolyte diffusion (18; 19), but it may not limit viscoelastic mechanisms involving solvent flow or unbound polyelectrolytes. Thus, the multilayer maintains a more orthotropic symmetry where the elastic modulus is an apparent $E_{\text {perp }}$ normal to the layer. Covalent bonding between polymer chains within the multilayer was done with EDC and Sulfo-NHS (see Methods) and increased the elastic modulus $\sim 15$-fold without a significant change in gel thickness. Stiffening from these covalent bonds implies that polymer chains in the un-crosslinked, ionic films are not so strongly bound to each other, mirroring the crosslink-dependent stiffness of PA gels.

\subsection{Layer-by-Layer Films: Collagen Addition and Cell Spreading}

To study cell spreading on these multilayers, we pre-adsorbed collagen-I onto the surface of both the native and crosslinked multilayers. Surface concentrations similar to PA gels that gave "optimal" spreading (Fig. 3B) were sought (i.e. $~ 50-1000 \mathrm{ng} / \mathrm{cm}^{2}$ ). This approximate range was achieved by overnight incubations and was verified, as with the PA gels, by use of calibrated imaging of fluorescent collagen (see Methods). Furthermore, FRAP (fluorescence recovery after photobleaching) experiments were done on crosslinked PLL/HA films with fluorescent collagen adsorbed. This was spotbleached under high laser power and then the intensity of the spot was followed. Collagen mobility was very limited, with recovery of bleached intensity saturating at $\sim 20 \%$ of bleached intensity over cell culture-relevant time scales ( hours). Thus the multilayer-bound collagen seems reasonably well adsorbed and at surface concentrations 
on both native and cross-linked multilayers that have already been found optimal for SMC spreading on PA gels (Fig. 3B).

SMCs were therefore plated on the collagen-coated multilayer films, and cell spreading was observed after 4 hours (Fig. 4). Spreading is clearly promoted on the much stiffer, crosslinked multilayers. Initial results for projected cell area are thus consistent in trend with the results above on PA gels (compare Fig. 3A to Fig. 4).

\subsection{Surface Probe Resolution on Elastic Gradients}

The results above are sensible and consistent only to the extent that the surface probe measurements of elasticity are well calibrated. While excellent correlation with macroscopic tension tests have been achieved using thick PA gel samples of $70 \mu \mathrm{m}(1)$, the spatial resolution limits for heterogeneous, soft samples have not yet been addressed. Tissues are, of course, non-homogeneous as well as soft, and so validated methods of measurement are important for present and future work in this general area of cell responses to elasticity. This section concludes with a final surface probe result on gradient PA gels.

A microfluidic mixing system created crosslinking gradients with fixed acrylamide monomer concentration (8\%) from several input ports of various bisacrylamide crosslinker concentrations. Through a series of channels, the mixer generates a smooth gradient in crosslinker concentration (from $0.048-0.48 \%$ ) that spans the width of the gel $(2.8 \mathrm{~mm})$ in a $90 \mu \mathrm{m}$ thick PA gel. Gradient gels $(\mathrm{n}=2)$ were probed using pyramid-tipped AFM probes, with measurements $(\mathrm{n}=157)$ made at multiple locations, $200 \mu \mathrm{m}$ apart along the width of the gel (Fig. 5). When compared to homogeneous PA 
gels of identical monomer concentration and indicated crosslinking concentration (inset square data, Fig. 5), the two input points can be clearly identified and an equal mixture of the two inputs (i.e. $1 / 2[0.048 \%+0.48 \%]=0.264 \%$ ) has a modulus found in the middle of the gel. A linear fit of the elastic gradient in the central portion of the gel yields a slope of $12 \mathrm{~Pa} / \mu \mathrm{m}$ for the detectable change in elastic modulus with distance. This result illustrates the potential accuracy of AFM-determined elasticities in non-homogeneous soft samples such as layered arterial cross-sections (eg. Fig.1).

\section{Discussion}

Tissues, in general, and arteries, in particular, are heterogeneous in their elasticity as well as anisotropic, soft, and non-linear (8). Surface probing here of sectioned hydrated tissues by AFM appears to be an accurate method to locally measure effective elasticities in the relevant kiloPascal range. Histological sectioning of a tissue can in principle compromise the tissue's mechanical integrity. However, comparisons seem very good with macroscopic measurements of moduli on larger and more intact SMC tissue samples (8), where surface effects are minimal. With care, the same AFM probing methodology can be applied to in vitro matrix mimics that range from micron thin films of covalently crosslinked gels, to physically crosslinked multilayers, and microgradient gels. 


\subsection{Probing the Elasticity of Soft Polymer Films with AFM}

Measuring the elasticity of polymeric films of various types may seem relatively trivial when compared to the complex structure, loading conditions, and viscoelasticity of tissues. However, in the thin film limit, substrate defects and rigid supports can prove a significant complication. Nonetheless, the correlation between macroscopic and nano-scale mechanics noted elsewhere (1) for hydrated gels carries over to thin film regimes. This proves to be only minimally affected by indenter geometry within this height range, since the moduli determined by sphere and blunted pyramid tips for $70 \mu \mathrm{m}$ gels is not statistically different $(\mathrm{p}=0.45)$. Using sphere-tipped probes, the elastic modulus did not significantly change as a function of height $(\mathrm{p}=0.38)$ in PA gels of high or low crosslinking density studied here (Fig. 2C). In many past applications of Hertz cone and sphere models $(21 ; 23-25)$, an implicit assumption had been that indentations were being made into a semi-infinite substrate regardless of a rigid support. As one approaches the limit of thin film elasticity, stiffening due to the rigid, constraining support and indenter geometry become important to substrate mechanics (Fig. 2C).

The few micron thick films of (PLL/HA) $)_{20}$ present further complications in probing by AFM. These films are heterogeneous in being athotropic, and they are noncovalent assemblies that can creep under the stress of the AFM probe. Moreover, in the native films, the outermost layers are likely to be more sparse and hydrated, with a reduced electrostatic interaction versus the more ordered layers below. Even in the lower layers though, fluorescent recovery after photobleaching demonstrates significant PLL fluidity as this polymer diffuses among and within layers $(18 ; 19)$. This suggests that 
water as well as crosslinking reagents will also readily diffuse into the layers. Converting the purely electrostatic crosslinking of such native films (ionic interactions) to covalent crosslinking between the ammonium groups of PLL and the carboxylate groups of HA (15) (amide bonds) leads to the not so surprising result that film height decreases as polymer chains in the soft upper layers are crosslinked and water is shed from the film. Increased layer density along with the covalent rather than physical bonds both contribute to the 10 -fold increase in the apparent elastic modulus, $E_{\text {perp }}$ (Fig. 4).

\subsection{Cell Spreading Responses and ECM Relevant Microenvironments}

Cell spreading on thin PA films of several microns appear equivalent to those reported recently on much thicker, soft or stiff PA substrates $(1 ; 2 ; 6 ; 29)$. The basic trend in projected cell area, given sufficient surface accessible collagen (Fig. 3B inset), is a hyperbolic, saturable spreading with increasing modulus, $E_{g e l}$. On the softest and thinnest PA gels examined ( $1 \mathrm{kPa} ; 5 \mu \mathrm{m}$ thick), spreading differences were minimal. This implies surface tractions generated by cells decay prior to reaching the rigid support.

With increasing stiffness, thin PLL/HA substrates qualitatively promoted spreading similar to PA gels at roughly similar collagen concentrations. The apparent elasticity scale, $E_{\text {perp }}$, for the multilayers should not, however, be equated with the $E_{g e l}$ for PA gels, which seem to be more ideal in their material behavior. For all systems studied, however, he AFM elasticity measurements were taken in seconds (at indentation rates of $\mu \mathrm{m} / \mathrm{sec}$ ), tending to make viscoelastic materials appear more elastic than viscous. Differences with the multilayers thus arise from slow polymer and collagen diffusion that is absent from even the most loosely crosslinked PA gels bearing covalently attached 
collagen. Crosslinked multilayers are stiffer, as expected, due to covalent crosslinks rather than electrostatic crosslinks, and this tends to promote cell spreading on a collagen coating. For future measurements, however, longer time scales of minutes to hours seem relevant to understand the properties seen by cells in spreading.

The hyperbolic form of spreading with increasing $E$ reveals a mechanical setpoint, $E_{1 / 2 \text {-spread, }}$ for spreading (1). This is expected to correspond to a typical in vivo stiffness of cell-matrix environments. Given dissimilar behavior in other cell types, such as neuronal branching (7) and endothelial tube formation (30) on soft gels, it is reasonable to assume that $E_{1 / 2 \text {-spread }}$ differs among cell types, which require wide-ranging micromechanical environments for different cell/tissue function. Further evidence is noted in the original studies of epithelial cells on soft gels (3) where cells were shown to have smaller, dynamically ruffled shapes, and fibroblasts were reported to be more polarized on soft gels. Nevertheless, matrix compliance and ligand density are coupled variables that determine cell responses dependent on the typical matrix stiffness in vivo $(1 ; 4)$. By explicitly measuring such a range here for vascular SMCs in arterial media by AFM indentation, we note that the elasticity range of $5-8 \mathrm{kPa}$ agrees with typical $E_{1 / 2 \text {-spread }} \sim$ $8-10 \mathrm{kPa}$.

Biphasic behavior seen with cell spreading versus collagen concentration on soft materials is mechanistically overlapping with biphasic behavior in cell migration versus ligand on rigid substrates (31). At low ligand densities, there is a lack of adequate attachment for a cell to pull itself forward (or spread as here), while at high ligand densities, detachment from ligand is needed to move forward (32). Detachment is probably not a limitation in cell spreading on gels, so recently introduced spreading 
models contain a penalty term for cell stretching (1). Extension is ficilitated by the actin cytoskeleton, which is signaled to assemble through cell adhesion to stiff substrates by signaling mechanisms that are under active investigation but do seem to involve calcium and Rho-family kinases $(2 ; 33)$. Whether cell spreading will force open membrane channels, will dissociate signaling complexes that initiate cascades, or will cause specific conformational changes in select proteins are all possible but unclear mechanisms at this point.

\subsection{Lateral Resolution of AFM on soft gels}

In addition to ligand concentration dependences, spatial variation in matrix elasticity is known to give rise to a form of cell motility termed durotaxis $(2 ; 5)$. Cells tend to move to stiffer substrates, with immobilization by spreading. While step and steep gradients are detectable by cells $(2 ; 5)$, whether or not shallower but clearly measurable gradients of $12 \mathrm{~Pa} / \mu \mathrm{m}$ (Fig. 5) can produce a durotactic response has yet to be reported. Although the gradient gels studied here were thick, the results for homogeneous gels (Fig. 2C) indicate that thinner gels with gradients should yield similar results.

\section{Conclusion}

Surface probe measurements of local elasticity allow a relatively accurate assessment of the mechanical properties of various soft matrices, materials, and thin films. Such assessments correlate well with adhesive spreading of a cell: stiffer materials tend to promote spreading of smooth muscle cells and half-max spreading 
occurs on PA matrices that approximate the stiffness of the SMC-rich arterial media. Remarkably, films that are microns thick and much thinner than the spread dimensions of a cell still prove equivalent to much thicker hydrated substrates. The answer likely lies in micron-scale or less lateral substrate displacements exerted by a cell; such tractions can decay sufficiently before the rigid support proves constraining. Current challenges in surface probe measurement lie in accurately obtaining highly localized determinations of not only elasticity but also of more complex rheology for natural and synthetic matrices especially on appropriate time scales that are biologically relevant. Surface or material preparation difficulties lie in the controlled generation of uniform, sub-micron, and soft gels either with PA gels, multilayers, or other materials.

\section{Acknowledgements}

The authors would like to thank Dr. Keith Gooch for kindly providing pig carotid arteries and Sooyoung Kim (Boston University) for preparing the gradient gels. Support was provided by grants or fellowships from the NIH, the NSF, the MDA, and the Ashton Foundation.

\section{References:}

[1] A. Engler, L. Bacakova, C. Newman, A. Hategan, M. Griffin, D. Discher, Biophys J 86 (2004) 617-628.

[2] C.M. Lo, H.B. Wang, M. Dembo, Y.L. Wang, Biophys J 79 (2000) 144-52.

[3] R.J. Pelham, Y.-L. Wang, Proc Natl Acad Sci U S A 94 (1997) 13661-13665.

[4] C. Gaudet, W.A. Marganski, S. Kim, C.T. Brown, V. Gunderia, M. Dembo, J.Y. Wong, Biophys J 85 (2003) 3329-35.

[5] J.Y. Wong, A. Velasco, P. Rajagopalan, Q. Pham, Langmuir 19 (2003) 1908-1913.

[6] C.A. Reinhart-King, M. Dembo, D.A. Hammer, Langmuir 19 (2003) 1573-1579.

[7] L.A. Flanagan, Y.E. Ju, B. Marg, M. Osterfield, P.A. Janmey, Neuroreport 13 (2002) 2411-5. 
[8] Y.-C. Fung, Biomechanics: Mechanical Properties of Living Tissues, New York, Springer, 1993.

[9] A.M. Collinsworth, S. Zhang, W.E. Kraus, G.A. Truskey, Am J Physiol Cell Physiol 283 (2002) C1219-27.

[10]C. Rotsch, K. Jacobson, M. Radmacher, Proc Natl Acad Sci U S A 96 (1999) 921-6.

[11] C. Rotsch, M. Radmacher, Biophys J 78 (2000) 520-35.

[12] A.B. Mathur, A.M. Collinsworth, W.M. Reichert, W.E. Kraus, G.A. Truskey, J

Biomech 34 (2001) 1545-53.

[13] G. Decher, J.D. Hong, J. Schmitt, Thin Solid Films 210-211 (1992) 831-5.

[14] G. Decher, Science 277 (1997) 1232-1237.

[15] L. Richert, P. Lavalle, D. Vautier, B. Senger, J.F. Stoltz, P. Schaaf, J.C. Voegel, C. Picart, Biomacromolecules 3 (2002) 1170-8.

[16] J.D. Mendelsohn, S.Y. Yang, J. Hiller, A.I. Hochbaum, M.F. Rubner, Biomacromolecules 4 (2003) 96-106.

[17] Y.-L. Wang, R.J. Pelham, Methods in Enzymology 298 (1998) 489-496.

[18]C. Picart, J. Mutterer, L. Richert, Y. Luo, G.D. Prestwich, P. Schaaf, J.-C. Voegel, P. Lavalle, P.N.A.S. 99 (2002) 12531-12535.

[19] L. Richert, F. Boulmedais, P. Lavalle, J. Mutterer, E. Ferreux, G. Decher, P. Schaaf, J.-C. Voegel, C. Picart, Biomacromolecules in press (2004).

[20] G. Hermanson, Bioconjuate Techniques, San Diego, Academic Press, 1996.

[21] J. Domke, M. Radmacher, Langmuir 14 (1998) 3320-5.

[22] E.K. Dimitriadis, F. Horkay, J. Maresca, B. Kachar, R.S. Chadwick, Biophys J 82 (2002) 2798-810.

[23]

[24]

[25]

[26] R.E. Mahaffy, C.K. Shih, F.C. MacKintosh, J. Kas, Physical Review Letters 85 (2000) 880-883.

[27] N. Wang, E. Ostuni, G.M. Whitesides, D.E. Ingber, Cell Motil Cytoskeleton 52 (2002) 97-106.

[28] J.L. Tan, J. Tien, D.M. Pirone, D.S. Gray, K. Bhadriraju, C.S. Chen, P.N.A.S. 100 (2002) 1484-1489.

[29] K.A. Beningo, M. Dembo, I. Kaverina, J.V. Small, Y.L. Wang, J Cell Biol 153 (2001) 881-8.

[30] C.F. Deroanne, C.M. Lapiere, B.V. Nusgens, Cardiovasc Res 49 (2001) 647-58.

[31] S.L. Goodman, G. Risse, K. von der Mark, J Cell Biol 109 (1989) 799-809.

[32] D.A. Lauffenburger, J.J. Lindermann, Receptors: Models for binding, trafficking, and signaling, London, Oxford University Press, 1996.

[33] A.D. Bershadsky, N.Q. Balaban, B. Geiger, Annu Rev Cell Dev Biol 19 (2003) 67795. 
Figure 1: Arterial Section. Arteries contain three layers of distinct composition and artery-dependent thickness. The Intima faces the vessel lumen and is lined with endothelial cells. The Media is both elastic and contractile with SMCs typically composing $\sim 30-60 \%$ of the mass (20). Collagen is the predominant matrix protein at $\sim 10-40 \%$ by mass, with elastin also being a significant fraction. The outermost, protective layer is the Adventitia, which is expected to be far stiffer.

Figure 2: Thin Film PA gels and their Elasticity. (A) Schematic of the polymerization process used to create PA gels down to micron-sized thickness. Acrylamide solutions containing a low concentration of appropriate spacer beads were polymerized between a lower aminosilinated coverslip and an upper chlorosilanized coverslip with a weight on top. Once fully polymerized, the non-stick upper coverslip was removed, yielding a thin PA gel. (B) Typical AFM indentation profile of $5 \mu \mathrm{m}$ thick PA gel using a microspheretipped probe. The fit used here is a thin film correction (22) of Sneddon's model of an axisymmetric probe indenting an infinite material. Agreement between fit and experiment is seen up to indentations of $\sim 50 \%$ in gel height. (C) PA thin film elasticity proves essentially independent of thickness as measured by AFM indentation. 5\% acrylamide solutions were mixed with either $0.3 \%$ (squares) or $0.03 \%$ (circles) bisacrylamide crosslinker and polymerized with beads of varying radius. Closed points use the thin-film correction to the Hertz sphere model; open points use the classic Hertz sphere model. The horizontal lines are averages of the thin-film corrected results. 
Figure 3: Cell Spreading on Thin PA Films. (A) Spreading of SMCs was assessed on $5 \mu \mathrm{m}$ and $70 \mu \mathrm{m}$ thick PA gels as well as glass. No statistical difference was noted between cell populations on gels of similar compliance but different thickness $(p=0.34)$. However, cell spreading shows a steep dependence on the elastic modulus of substrates with similar collagen density, as observed before (1). Inset images show representative cells spreading on both 'soft' $1 \mathrm{kPa}$ and 'very stiff' $35 \mathrm{kPa}$ PA gels. White arrows in the images indicate $5 \mu \mathrm{m}$ diameter spacer beads used to set the gel thickness. Scale bar $=50$ $\mu \mathrm{m}$. (B) $70 \mu \mathrm{m}$ PA gel substrates of varying compliance and glass were coated with various collagen densities. While cells within the optimal region behaved similarly to those above, cells on both higher and lower collagen concentrations were smaller, indicating a biphasic behavior $(1 ; 4)$ dependent on the elasticity of the substrate. Inset image shows quantum dot labeling of a collagen-coated $8 \mathrm{kPa}$ PA gel. Scale bar $=15$ $\mu \mathrm{m}$.

Figure 4: Cell Spreading on thin (PLL/HA) $)_{20}$ Films. Thin multilayer films of poly(Llysine)/ hyaluronic acid (PLL/HA)20, assembled by a layer-by-layer method (18) and selectively crosslinked, were assessed by AFM indentation. Multilayers were subsequently coated with a collagen monolayer to promote cell adhesion. Cells were then plated on these multilayer films ( 4 $\mu \mathrm{m}$ thick), and the spread cell area was measured. Cells spread very little on un-crosslinked films, and yet chemically crosslinked (PLL/HA) 20 films, with a 15-fold increase in elastic modulus, showed a 5fold increase in cell spread area. Cell morphology resembled that on PA gels. 
Surface Science - Revised Manuscript

Figure 5: PA gels with Resolvable Elasticity Gradients. Using techniques described elsewhere (5), a 10-fold crosslinker gradient was established across the width of an $8 \%$ PA gel by microfluidic mixing of solutions containing $0.48 \%, 0.264 \%$, and $0.048 \%$ bisacrylamide. Using AFM indentation combined with lateral stage displacements, the elastic modulus was locally measured and mapped across the gel. Binned data is displayed as a function of length across the gel. Open squares denote gel standards containing the indicated crosslinker concentration. These correspond to the microfluidic inputs of minimum (left), intermediate (center), and maximum (right) crosslinker concentrations.

Table 1: Local elasticity of medial layer in arterial section as determined by AFM.

\begin{tabular}{|l|l|l|}
\hline AFM Indenter & Fitted Depth & Apparent $E_{\text {media }}$ \\
\hline Sphere-tipped $(\mathrm{n}=25)$ & $2.5 \pm 0.4 \mu \mathrm{m}$ & $5.7 \pm 0.3 \mathrm{kPa}$ \\
\hline Pyramid-tipped $(\mathrm{n}=42)$ & $2.7 \pm 0.2 \mu \mathrm{m}$ & $7.3 \pm 0.7 \mathrm{kPa}$ \\
\hline
\end{tabular}




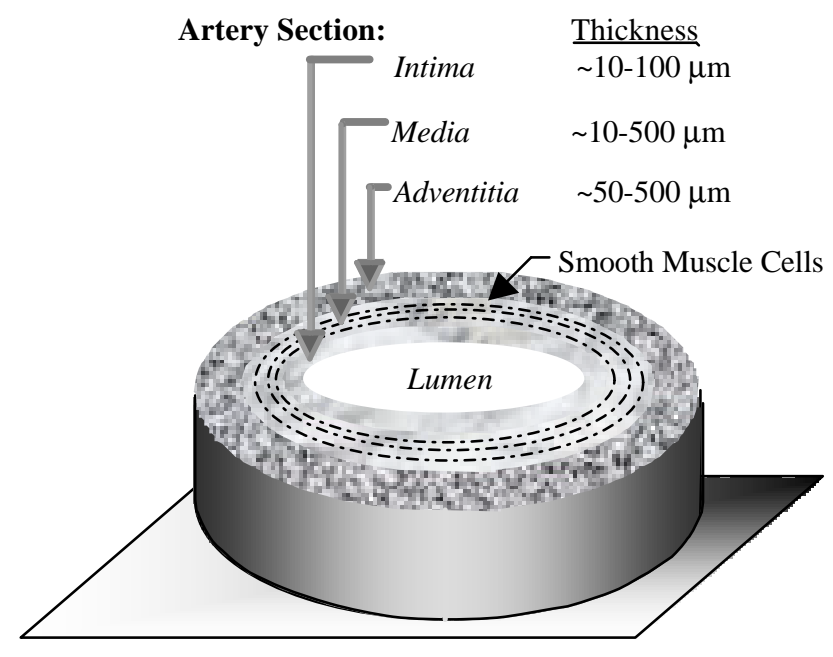


A

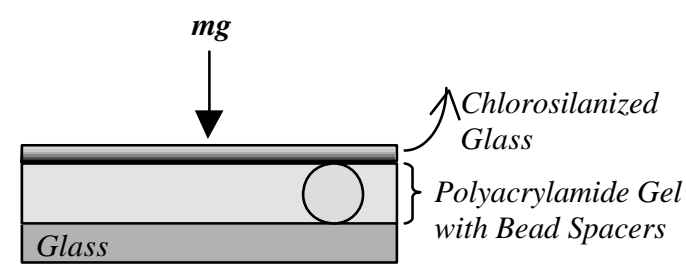

B
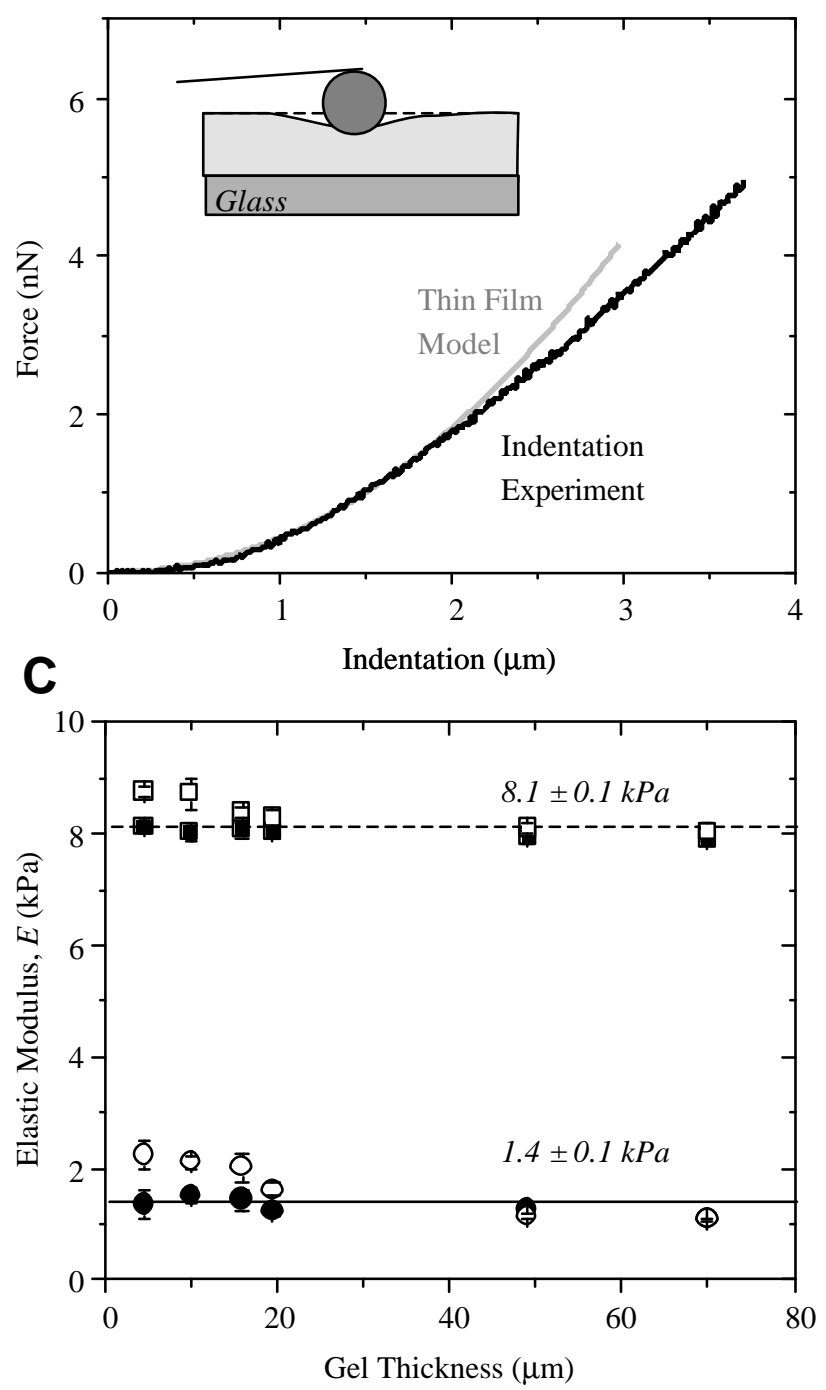

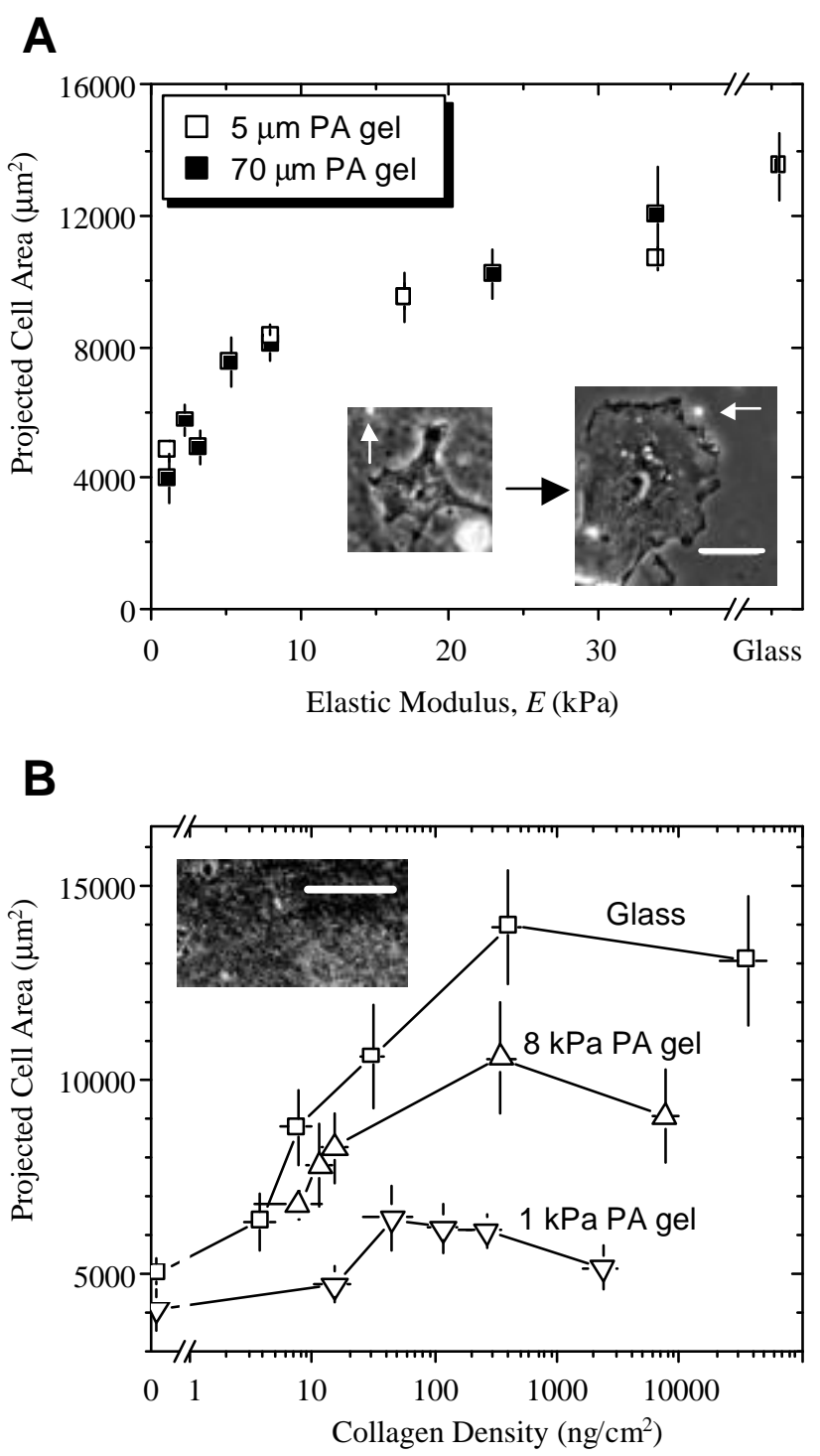
Figure 4

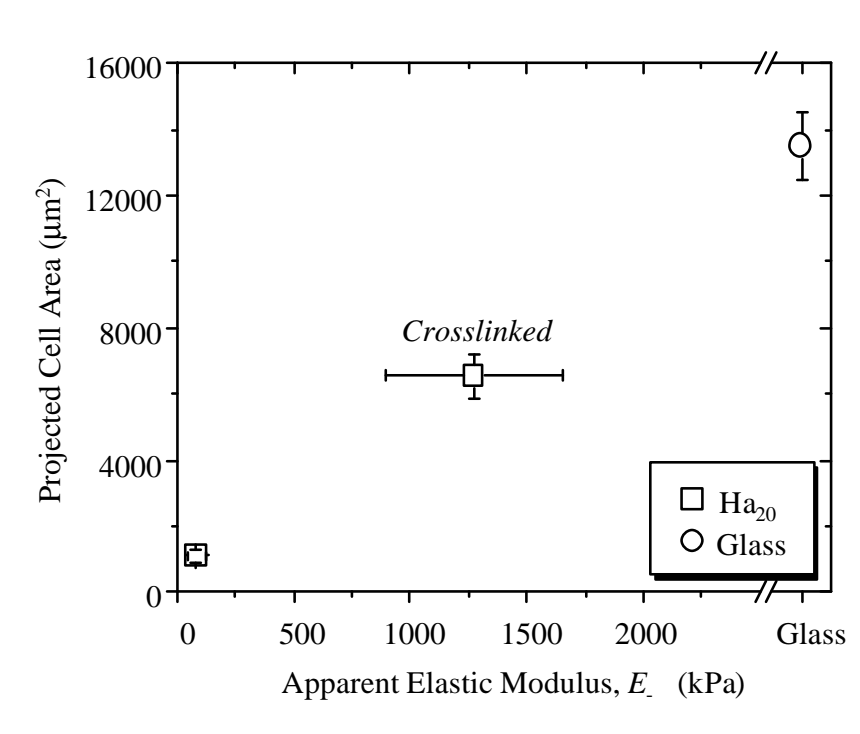

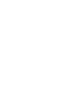




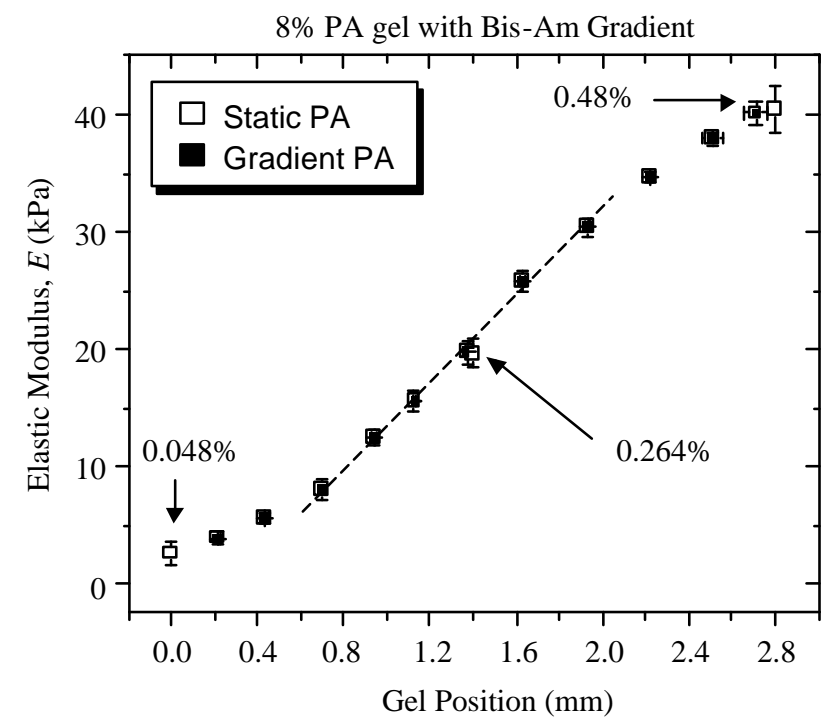

